\title{
COMPARACIÓN DE TÉCNICAS DE EVALUACIÓN MULTICRITERIO EN SISTEMAS DE AYUDA A LA DECISIÓN ESPACIAL PARA LA LOCALIZACIÓN ÓPTIMA DE USO RESIDENCIAL
}

\author{
María Teresa Lamelas Gracia \\ Centro Universitario de la Defensa de Zaragoza, Academia General Militar \\ Ctra. de Huesca s/n. 50090, Zaragoza, Spain \\ tlamelas@unizar.es
}

\begin{abstract}
Resumen: Los Sistemas de Ayuda a la Decisión Espacial (Spatial Decision Support System, SDSS) han resultado ser extremadamente útiles para resolver los conflictos, entre los diferentes usos del suelo, que aparecen comúnmente en el proceso de ordenación sostenible del territorio. Este artículo presenta una comparación de los resultados obtenidos de los análisis de la idoneidad del terreno para acoger un uso residencial, aplicando dos técnicas de análisis multicriterio diferentes. Los análisis multicriterio se han llevado a cabo en un entorno ráster con dos objetivos, el análisis de localización óptima y la búsqueda o selección de alternativas óptimas. En el primer caso, cada pixel en la zona de estudio es considerado una alternativa de localización. Para este análisis se ha aplicado el método sumatoria lineal ponderada (SAW), que es fácilmente integrable en SIG. En el segundo caso, se ha hecho uso de la metodología PROMETHEE-2 y una serie de alternativas de localización previamente establecidas. A partir de los resultados, la principal conclusión es la similitud en los modelos obtenidos al aplicar diferentes metodologías y la necesidad de aplicar aproximaciones estocásticas para tener control de la incertidumbre en los datos de entrada.
\end{abstract}

Palabras clave: Ordenación del territorio, SDSS, SAW, AHP, PROMETHEE, desarrollo sostenible.

Abstract: The development of spatial decision support systems (SDSS) has greatly assisted efforts for solving the complex land-use conflicts that com- 
monly appear in a sustainable land-use management. In this paper we compare the results obtained by the application of two distinctive landuse suitability analyses to the location of residential land use, applying two different multicriteria analysis techniques. The multicriteria analysis employed has been performed in a raster environment and been used for two objectives, namely for site search and a spatial suitability assessment. During the site search analysis each pixel was considered a potential location alternative. This analysis was performed using a simple additive weighting (SAW) method, which can easily be performed in GIS. A site selection analysis was then performed using the PROMETHEE- 2 methodology and using a set of predefined alternatives. The main conclusion from the obtained results is the similarity of the results obtained after applying the different approaches and the necessity of applying stochastic approaches in order to control the uncertainty of the input data.

Keywords: Land-use management, SDSS, SAW, AHP, PROMETHEE, sustainable development.

\section{Introducción}

\subsection{Antecedentes generales}

La legislación europea promueve un desarrollo sostenible bien planificado que incluya tanto la dimensión económica, como la social y medioambiental. No obstante, uno de los importantes desafíos del siglo XXI es conseguir el desarrollo sostenible en las grandes ciudades.

En los años setenta y ochenta, escasamente se mencionaba en la bibliografía científica el desarrollo sostenible en ciudades (McGranahan y Satterthwaite, 2003; Mitlin y Satterthwaite, 1996). Con posterioridad a la Declaración de Río de Naciones Unidas en 1992 (http://www.un.org) y a la Agenda 21 y su programa adicional de implementación, la promoción de un desarrollo sostenible en los establecimientos humanos ha suscitado una creciente preocupación. De hecho, en los años noventa, se organizaron varias conferencias internacionales abordando el asunto de la urbanización rápida, su desarrollo y administración, y el término "Mega-ciudad" fue ampliamente difundido.

En la Estrategia Temática para el Medio Ambiente Urbano de la Unión Europea se recalca el importante papel de las zonas urbanas en el cumplimiento de los objetivos de la Estrategia de la Unión Europea para un Desarrollo Sostenible, ya que es en las ciudades donde se concentran muchos problemas medioambientales, pero también son el principal motor económico. 
El objetivo general debería ser mejorar o cambiar la organización en el proceso de toma de decisiones, para que la consideración de asuntos socioeconómicos y medio ambientales se pueda integrar completamente en el mismo y se asegure la participación pública.

Los Sistemas de Información Geográfica (SIG) son enormemente necesarios para cumplir las funciones de Ordenación del Territorio, ya que los instrumentos a utilizar deben ser actualizables, multi-escalares y deben contener una amplia gama de informaciones relativas al medio ambiente; es decir, al medio físico, medio biótico, aspectos antrópicos y sus interrelaciones (Amadio et al., 2002).

El desarrollo de Sistemas de Ayuda a la Decisión Espacial (Spatial Decision Support System, SDSS) en los últimos años, ha constituido una ayuda importante en la resolución de conflictos entre los diferentes usos del suelo, que aparecen comúnmente en el proceso de ordenación sostenible del territorio.

Los SDSS han sido definidos como la combinación de metodologías sofisticadas para el apoyo a la toma de decisiones (técnicas de evaluación multicriterio - EMC) y Sistemas de Información Geográfica - SIG (Gómez-Delgado y Barredo, 2005; Jankowski, 1995; Malczewski, 2004; Marinoni, 2005), y por esta razón son herramientas óptimas para el planeamiento y desarrollo sostenible de zonas urbanas.

Aunque la consagración del análisis multicriterio se realiza a partir de los años 70, es a partir aproximadamente del año 1991 (Gómez-Delgado y Barredo, 2005), en la denominada cuarta etapa de evolución en los SIG, cuando se desarrollan los análisis de decisión. Los trabajos de Voogd (1983), Janssen y Rietveld (1990), Carver (1991), Can (1993), Pereira y Duckstein (1993), Barredo y Bosque (1995), Bosque et al. (1999), Thill (1999), Eastman et al., (1993), Bosque y García (2000), Bosque y Moreno (2004), Moreno y Bosque (2010), entre mucho otros, indican la tendencia de integración de técnicas de Evaluación Muliticriterio (EMC) y SIG, tratando de solventar de algún modo las carencias analíticas de los SIG (Gómez-Delgado y Barredo, 2005).

La mayoría de los autores coincide en que las reglas aditivas de decisión (Sumatoria lineal ponderada, Análisis de la concordancia, Jerarquías analíticas, etc.) son los métodos para la toma de decisión multiatributo más conocidos y ampliamente utilizados en el proceso de toma de decisiones basado en la utilización de SIG (Gómez-Delgado y Barredo, 2005, Jankowski, 1995; Malczewski, 1999; Pereira y Duckstein, 1993; Vincke, 1986; Voogd, 1983).

Sin embargo, la integración de estas técnicas continúa generando ciertos problemas o dificultades en el momento de llevar a cabo aplicaciones específicas. Entre los inconvenientes más notables se pueden destacar los siguientes (Gómez-Delgado y Barredo, 2005): 
- La imposibilidad de aplicar técnicas de comparación por pares como PROMETHEE con largas series de datos debido a las limitaciones impuestas por los sistemas informáticos.

- La dificultad de implementación de determinados métodos que dificultan el análisis de los resultados, así como el desconocimiento del procedimiento metodológico interno por parte de los usuarios no especializados.

- La necesidad de generar software de procesamiento de datos integrado en SIG y basado en algoritmos que describen los métodos de EMC, lo que implica que la accesibilidad por parte de los usuarios a estos métodos es reducida.

\subsection{Antecedentes del proyecto}

En la periferia de Zaragoza, debido al rápido desarrollo urbano sufrido en las últimas décadas, las interacciones con el medio físico han sido, en su mayoría, ignoradas, aunque sea precisamente la disponibilidad de recursos naturales, como es el caso de la reserva de agua que proporciona el acuífero cuaternario sobre el que se asienta la ciudad, la causante del rápido desarrollo de la misma.

Una consecuencia del desarrollo no planificado y no sostenible, ha sido la ocupación por parte de espacios residenciales, industriales, comerciales, etc. de suelos de gran fertilidad, imposibilitando su uso agrícola, o sobre zonas de alto valor ecológico, afectándolas de forma negativa e irrecuperable (Lamelas, 2007). Del mismo modo, la intensificación del regadío ha provocado la subsidencia del terreno, que, en definitiva, ha producido daños y/o destrucción en carreteras, edificios y redes de abastecimiento de gas y agua (Soriano y Simón, 1995).

Desde nuestro punto de vista, la toma de decisiones sobre los usos del suelo es, en sí mismo, un problema de decisión multicriterio. Consideramos que los métodos de EMC son válidos para apoyar al proceso de toma de decisiones por medio del análisis de la idoneidad del terreno para acoger diferentes usos del suelo. Por esta razón, se inició un proyecto cuyo principal objetivo es el desarrollo de un esquema metodológico que facilitara la toma de decisiones sobre diferentes formas de uso del suelo, teniendo en cuenta aspectos geocientíficos, y posibilitando el desarrollo sostenible de un entorno urbano en crecimiento.

El propósito era llevar a cabo un análisis de la idoneidad o capacidad del terreno para acoger determinados usos del suelo, mediante la utilización de SDSS, con objeto de identificar su localización más apropiada para el futuro, de acuerdo con las preferencias específicas del desarrollo sostenible que implican la integración de aspectos socioeconómicos y medioambientales. Sin embargo, aunque las decisiones en el uso del 
suelo se hacen generalmente teniendo en cuenta criterios económicos y sociales, los aspectos geocientíficos no son, con frecuencia, considerados, o bien son considerados como criterios de menor importancia. No obstante, toda utilización del territorio que se planee desde la perspectiva del desarrollo sostenible -especialmente en comunidades en crecimiento- debe prestar atención a los geo-potenciales, que incluye a todos los georrecursos y georriesgos pertinentes.

El primer paso es, por tanto, la detección, descripción y modelización de georrecursos y georriesgos con la ayuda de SIG y técnicas de modelización bidimensionales y tridimensionales que serán integrados como mapas-criterio en un posterior análisis de la idoneidad del terreno para acoger un determinado uso del suelo.

Desde el punto de vista geológico, la Depresión del Ebro está limitada por los relieves pirenaicos, ibéricos y costero-catalanes, conformando un perímetro triangular. En el sector central de la depresión, desde el Oligoceno hasta el Mioceno, fueron depositados grandes cuerpos evaporíticos sedimentarios, especialmente en áreas con mayor subsidencia. En el curso del Cuaternario, las terrazas y glacis se han ido depositando en el techo de estas formaciones evaporíticas, conformando un acuífero aluvial que hace de la karstificación un proceso activo y permanente (Soriano y Simón, 1995). Por este motivo uno de los georriesgos a tener en consideración es la susceptibilidad a la aparición de dolinas.

A pesar de que el Río Ebro en su sector central, presenta un canal meandriforme más propenso a inundaciones, las continuas modificaciones de sus márgenes, así como su regulación mediante embalses y presas, han provocado un descenso en el número y magnitud de las inundaciones en las últimas décadas (Ollero, 1996). No obstante, en febrero de 2006 se produjo una crecida de hasta $3.000 \mathrm{~m} 3 / \mathrm{s}$ que inundó una gran superficie de terreno evidenciando la necesidad de tener en consideración este georriesgo en cualquier proceso de planificación.

Otro importante georriesgo en la zona de estudio es la erosión del suelo. Además, a causa de la gran solubilidad de los yesos, la erosión del suelo puede afectar negativamente a la calidad del agua, ya que el material es transportado por el flujo superficial incrementando su conductividad y salinidad, de manera que, desde el punto de vista ecológico, tanto la desertificación como la salinización son un problema en el entorno de Zaragoza (Machín and Navas, 1998).

Además, es importante considerar al suelo como un recurso agrícola, ya que los suelos pueden ser utilizados para muy diversos propósitos agrícolas, siempre y cuando se les apliquen los insumos necesarios. Sin embargo, debido a las características semiáridas de la zona de estudio y a la escasa cubierta vegetal, los suelos presentan un bajo contenido en materia orgánica y son muy frágiles. Por lo que de acuerdo con de la Rosa et al. (2004), el principal objetivo en la protección del suelo debe ser minimizar el coste 
medioambiental y socioeconómico que habitualmente produce el incremento en la productividad de los suelos mediante la predicción de su inherente capacidad para acoger un uso agrícola durante un largo periodo de tiempo.

Finalmente, el paisaje también debe considerarse como un recurso importante, no solo desde el punto de vista del observador, como recurso económico, sino también por su valor científico ya que integra hábitats esenciales para la conservación de especies, que en algunos casos se encuentran en peligro de extinción.

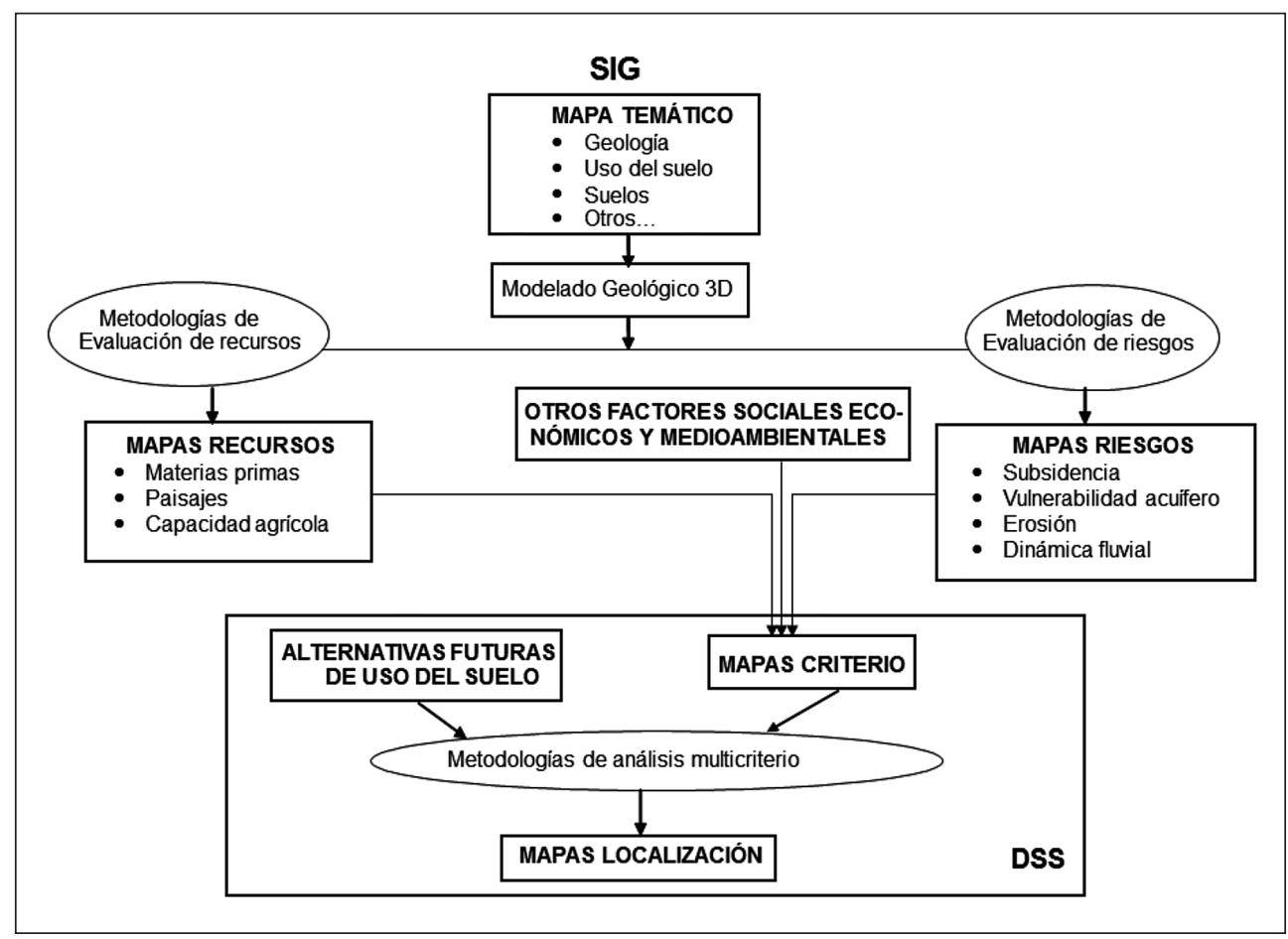

Figura 1. Esquema metodológico del proyecto.

Este artículo se centra en la última parte del proyecto, el análisis de la capacidad del terreno para acoger diferentes usos del suelo. Se comparan los resultados obtenidos tras llevar a cabo dos análisis distintos de la capacidad o idoneidad del terreno para acoger un uso residencial, aplicando diferentes técnicas de análisis multicriterio. El análisis multicriterio se ha llevado a cabo en un entorno ráster con dos objetivos bien diferenciados. En el primero de ellos, análisis de localización óptima, cada pixel en la zona de estudio representaba una posible alternativa de localización. Este análisis se 
ha desarrollado utilizando la metodología sumatoria lineal ponderada (SAW) que puede ser fácilmente implementada en SIG (Lamelas, 2007, 2009; Lamelas et al., 2006, 2008a, 2010). En el segundo objetivo, análisis de alternativa óptima, se ha utilizado la metodología PROMETHEE-2 (Brans et al., 1986) y una serie de alternativas de localización previamente establecidas (Lamelas, 2007). Las diferentes técnicas utilizadas en el proyecto han sido desarrolladas e integradas en ArcGIS por Marinoni (2004, 2005). Para más información acerca del esquema general del proyecto y de la modelización de georrecursos y georriesgos se pude consultar Lamelas et al. (2006, 2007, 2008b, 2009 y 2010) y Lamelas (2007, 2007b, 2009).

\section{Metodología}

En el contexto del análisis de la idoneidad o capacidad del terreno para determinados usos del suelo es importante diferenciar entre el problema de la selección de alternativa óptima (site selection problem) y el problema de búsqueda de localización óptima (site search problem). El propósito del análisis de selección de alternativa óptima es identificar la mejor localización para una determinada actividad, dado un grupo de localizaciones posibles. En el caso de que no exista un grupo determinado de alternativas, el problema se define como un análisis de localización óptima (Malczewski, 2004). Para llevar a cabo las dos aproximaciones, se deben seguir los siguientes pasos, que determinan en gran medida el desarrollo del presente apartado (figura 2):

- Definición de alternativas: posibles localizaciones.

- Definición de restricciones: áreas con restricciones para un determinado uso.

- Definición de los criterios: factores que tienen un papel importante en el proceso de decisión.

- Asignación de pesos de decisión.

- Aplicación de la metodología EMC.

\subsection{Análisis de búsqueda de localización óptima para uso residencial}

En este análisis cada pixel de la zona de estudio es considerado como una alternativa de localización. Las restricciones para la localización, se refieren a zonas en las que el uso residencial no está permitido. Estas restricciones de uso se deben en algunos casos a la existencia de otros usos (p.ej. uso industrial) y en otros casos a la existencia de protecciones medioambientales o restricciones de uso debidas a la planifi- 


\section{DEFINICIÓNDE}

ALTERNATIVAS

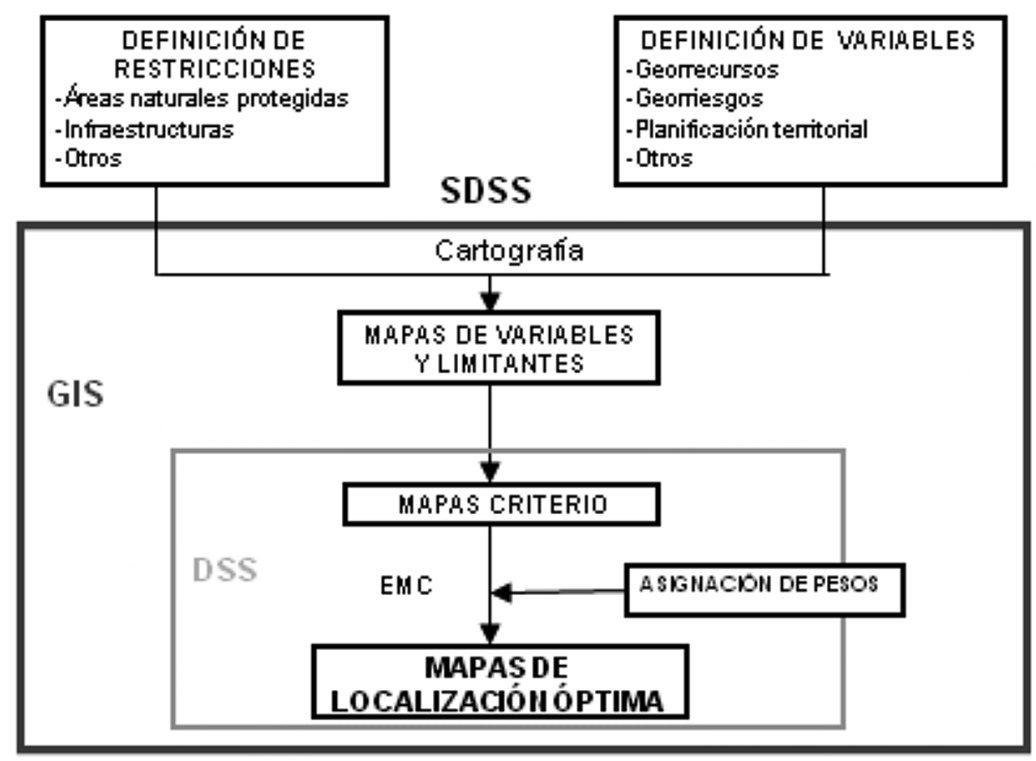

Figura 2. Pasos a seguir para el análisis de localización óptima de un determinado uso.

cación del territorio. Las restricciones de uso en la zona de estudio son las siguientes (figuras 3 y 4 ):

- Espacio Natural Protegido: Reserva Natural de los Galachos de La Alfranca, Pastriz, La Cartuja y El Burgo de Ebro, en la que está restringido el uso urbano. Cartografía proporcionada por el Gobierno de Aragón.

- Espacios urbanizados: cartografiados a partir del Mapa Topográfico Nacional (MTN), a escala 1:25.000, del Instituto Geográfico Nacional (IGN), actualizado mediante digitalización de fotografía aérea.

- Infraestructuras y sus áreas de protección: carreteras, vías de tren, canales, etc. Cartografiadas a partir del MTN a escala 1:25.000 y actualizadas.

- Planificación: Plan General de Ordenación Urbana de Zaragoza (PGOUZ) y Plan de Ordenación de los Recursos Naturales (PORN) del Ebro. Las zonas en las que existen restricciones de uso residencial se ha obtenido de cartografías proporcionadas por el Ayuntamiento de Zaragoza y el Gobierno de Aragón, respectivamente. 
- Vías pecuarias: reguladas y protegidas por la LEY 10/2005, de 11 de noviembre, de Vías Pecuarias de Aragón y cartografiadas a partir de información proveniente del Gobierno de Aragón.

- Polígonos industriales: zonas ya urbanizadas cuya información proviene del Instituto Aragonés de Fomento (IAF) del Gobierno de Aragón.

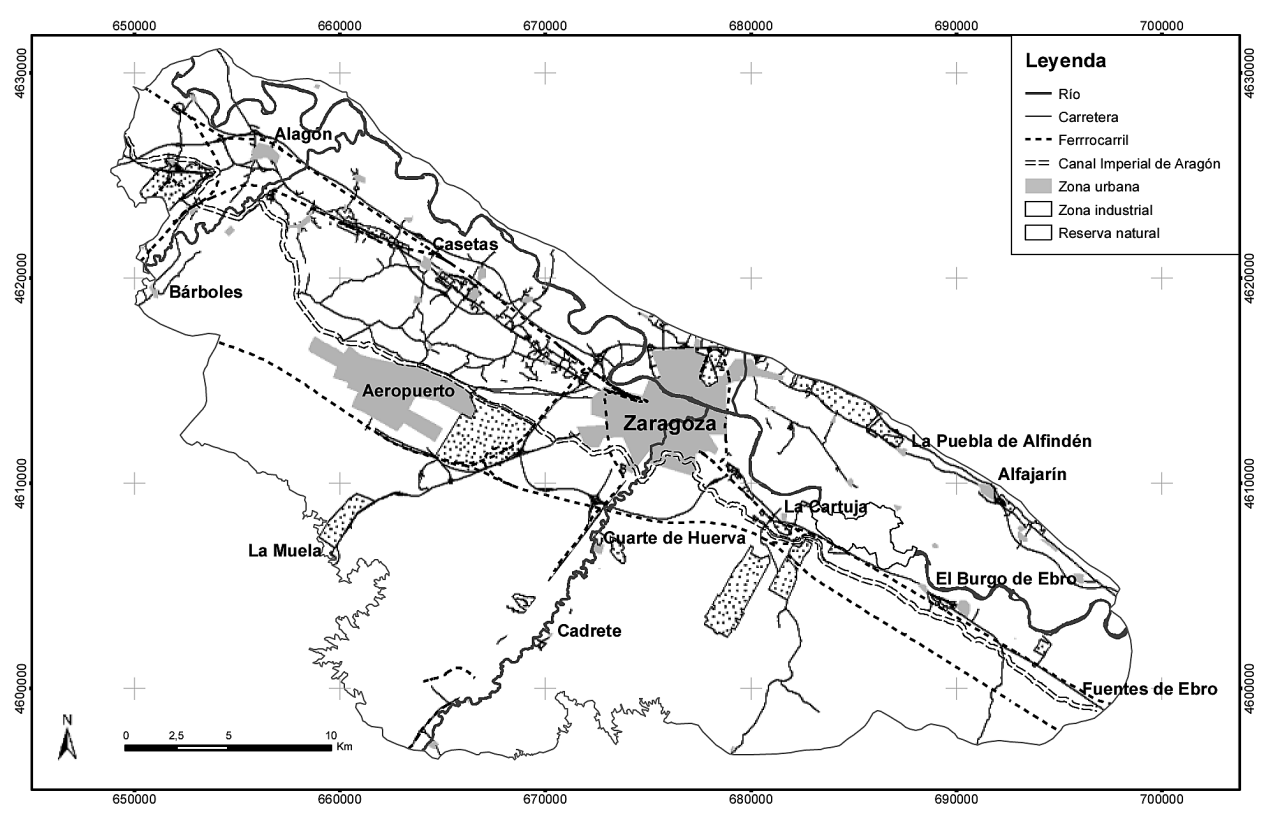

Figura 3. Restricciones para uso residencial.

Se tuvieron en consideración una gran variedad de factores sociales, económicos y medioambientales, ya que nuestro principal objetivo es el promover un desarrollo sostenible. La figura 5 muestra la cartografía de todas las variables. Estas variables son:

- Localización de espacios naturales de importancia: Zonas de Especial Protección para las Aves (ZEPAS) designadas por la Directiva 79/409/CEE del Consejo, de 2 de abril de 1979, relativa a la conservación de las aves silvestres, Hábitats designados por la Directiva 92/43/CEE del Consejo, de 21 de mayo de 1992, relativa a la conservación de los hábitats naturales y de la fauna y flora silvestres, Puntos de Interés Geológico incluidos en el inventario del Gobierno de Aragón y otras áreas naturales cuya cartografía ha sido cedida, principalmente, por el Gobierno de Aragón. Se considera que debe evitarse la afección a estas zonas, debido al 


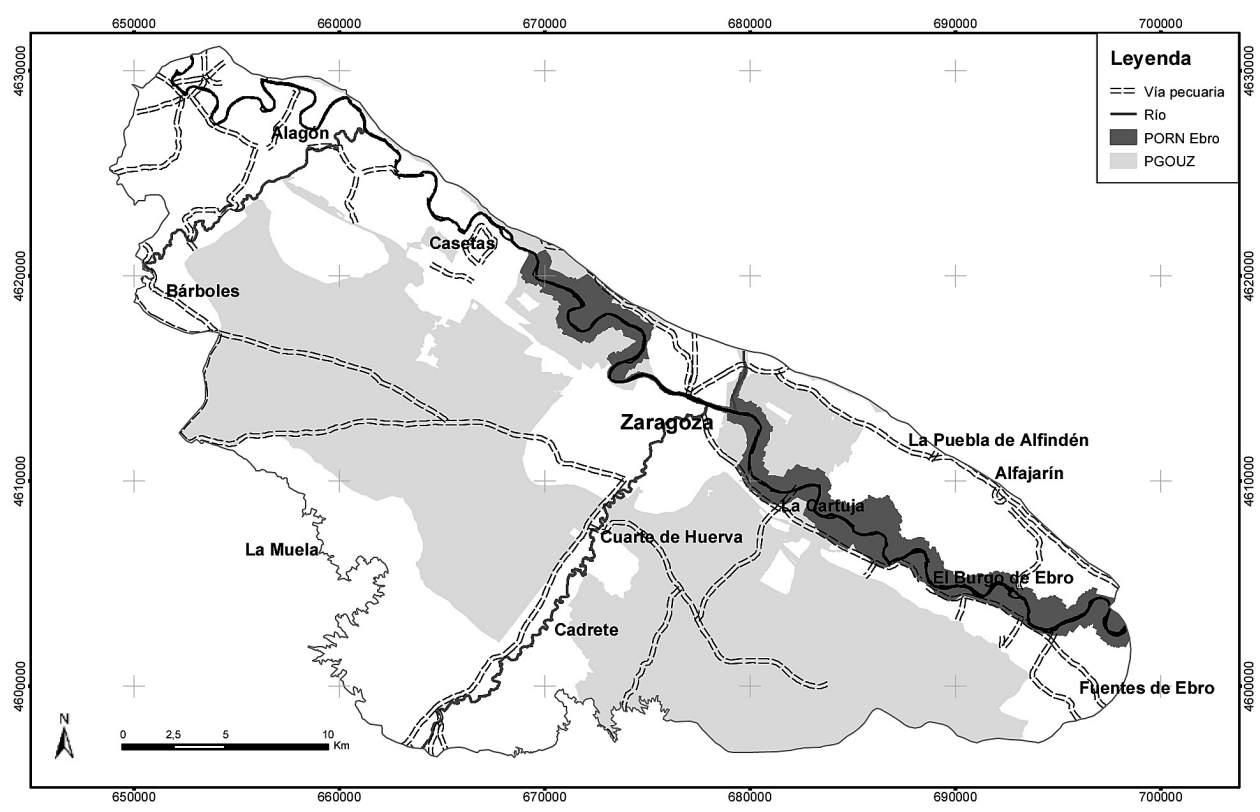

Figura 4. Restricciones para uso residencial (continuación).

gran valor de estos paisajes, no solo desde el punto de vista perceptual y económico, sino también desde el punto de vista científico.

- Susceptibilidad del terreno al desarrollo de dolinas: modelización realizada en el proyecto mediante la técnica de la Regresión Logística (Lamelas, 2007, 2007b; Lamelas et al., 2008a). Se considera que debe evitarse la construcción en zonas de elevada susceptibilidad a la aparición de dolinas como consecuencia de los procesos de karstificación existentes en la zona de estudio.

- Riesgo de inundación: cartografiado a partir de la georreferenciación y digitalización de los mapas analógicos elaborados por Ollero (1996). Las zonas con mayor riesgo de inundación deben de evitarse.

- Capacidad agrícola de los suelos: cartografía realizada para el proyecto mediante la implementación del Modelo Cervatana (C.S.I.C., 1996; de la Rosa y Magaldi, 1982; de la Rosa et al., 2002, 2004) en SIG (Lamelas, 2007, Lamelas et al., 2009). Se trata de un modelo cualitativo para evaluar la capacidad general agrícola de los suelos que utiliza los siguientes factores: relieve, suelos, clima y cubierta del suelo. Este factor se ha incluido ya que se considera que los suelos de mayor fertilidad deben ser conservados para su uso agrícola. 

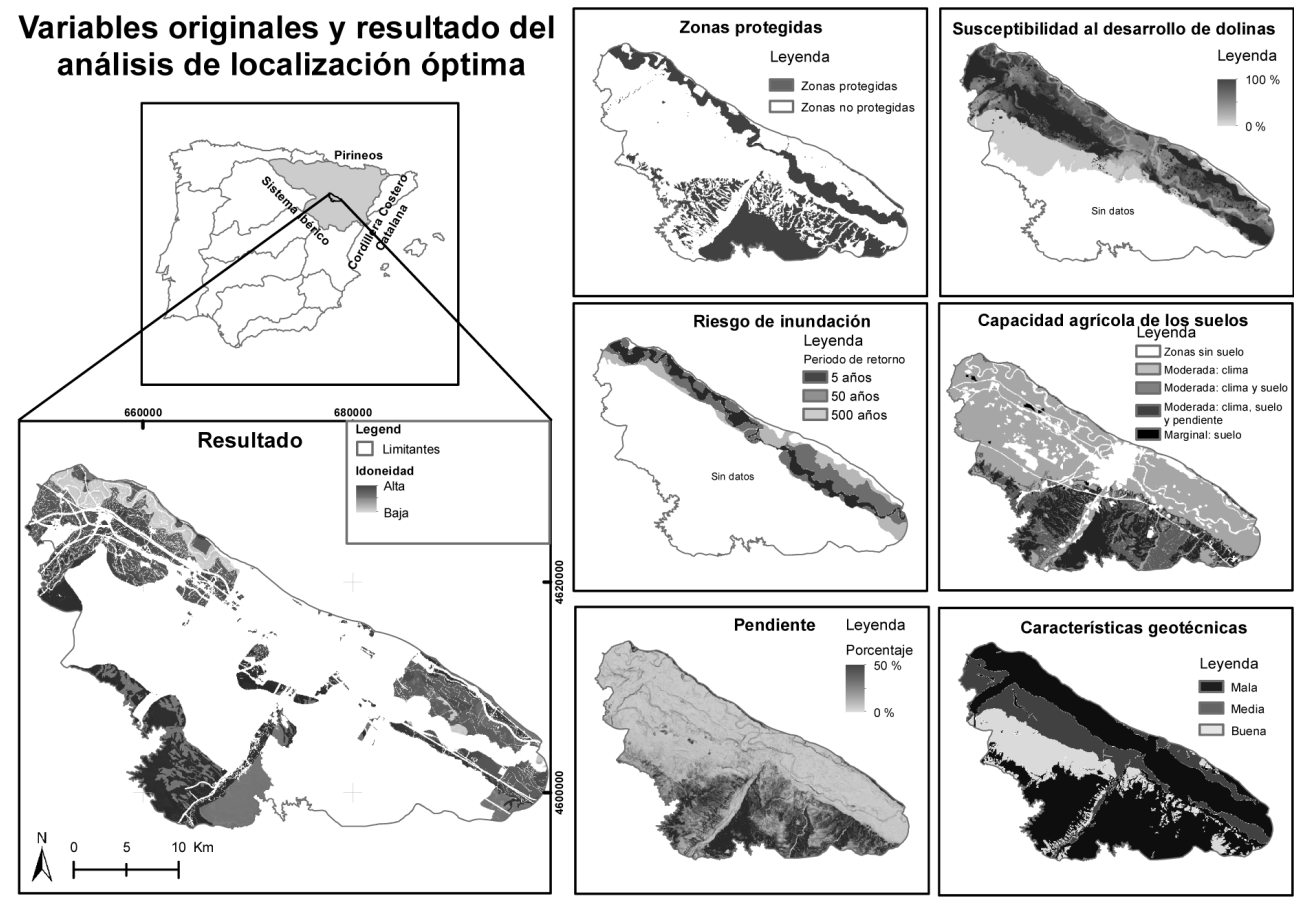

Figura 5. Cartografía de variables y resultado del análisis de búsqueda de localización óptima.

- Pendiente del terreno: desarrollada a partir del Modelo Digital de Elevaciones (MDE), resolución 20x20 m, del Ministerio de Agricultura (SIG Oleícola). Este factor se ha incluido ya que la pendiente incrementa o reduce la idoneidad del terreno para ser construido. Es un factor que influye en los desmontes necesarios para la explanación del terreno que tienen consecuencias tanto económicas como medioambientales.

- Caracterización geotécnica del subsuelo: modelo desarrollado en el proyecto a partir del Mapa Geológico a escala 1:50.000 (serie MAGNA) del Instituto Geológico y Minero de España (IGME), según las indicaciones del PGOUZ.

Muchas de las metodologías de EMC requieren que las variables sean estandarizadas para su incorporación como criterios en el proceso de localización óptima. Esta estandarización asegura el que los pesos de los criterios reflejen apropiadamente su importancia. En nuestro caso, el método de estandarización utilizado puede incluirse en la aproximación de escalas subjetivas (Malczewiski, 2004) ya que las variables se clasifican de acuerdo a unos rangos subjetivos. Estos rangos se determinan siguiendo, en algunos casos, las indicaciones de las Leyes medioambientales o, en otros casos, los 
rangos ya establecidos en los modelos desarrollados en el proyecto. De esta forma, las variables se han clasificado en 6 categorías: valores de 1 a 3 representan los peores valores de idoneidad, mientras que los valores de 4 a 6 representan los más óptimos. En las tablas 1-6 se puede consultar los valores asignados a los criterios en función de los valores originales. Para más información acerca del proceso de estandarización se puede consultar (Lamelas, 2007,2009; Lamelas et al., 2008a y 2010).

Uno de los problemas y críticas en la utilización de técnicas multicriterio es la necesidad de asignar pesos a los criterios. Existen una gran variedad de aproximaciones para llevar a cabo dicha asignación (Malczewski, 1999), aunque probablemente la más conocida es el método Jerarquías analíticas (Analytical Hierarchy Process, AHP).

Esta técnica requiere la comparación por pares de criterios, donde es preciso asignar valores numéricos que expresen la preferencia de un criterio frente a otro. Con posterioridad, los valores asignados son sintetizados para determinar el orden de preferencia de los criterios en términos de valores numéricos que equivalen a los pesos de los criterios. Finalmente, se ha de calcular el ratio de consistencia (CR) para valorar la consistencia de la matriz de comparación de criterios. Aplicaciones empíricas sugieren que este método de comparación por pares es una de las técnicas más efectivas para la toma de decisiones espaciales basadas en la utilización de SIG (Eastman et al., 1993; Malczewski et al., 1997). Existen una gran cantidad de ejemplos bien documentados de la aplicación de este método con éxito (Banai, 1993; Banai-Kashani, 1989; Miller et al., 1998).

Tabla 1. Valores utilizados en la estandarización de las zonas protegidas

\begin{tabular}{|l|c|}
\hline Valor original & Valor estandarizado \\
\hline Zonas dentro del perímetro de delimitación del espacio natural & 1 \\
Resto de zonas & 6 \\
\hline
\end{tabular}

Tabla 2. Valores utilizados en la estandarización de la susceptibilidad al desarrollo de dolinas

\begin{tabular}{|l|c|}
\hline Valor original & Valor estandarizado \\
\hline$>0.06$ & 1 \\
$0.04-0.06$ & 2 \\
$0.02-0.04$ & 3 \\
$<0.02$ & 5 \\
Fuera del modelo & 6 \\
\hline
\end{tabular}

En este caso los valores originales coinciden con los rangos de la modelización realizada mediante la técnica de la Regresión Logística (Lamelas, 2007, 2007b; Lamelas et al., 2008b). 
Tabla 3. Valores utilizados en la estandarización del riesgo de inundación

\begin{tabular}{|l|c|}
\hline Valor original & Valor estandarizado \\
\hline Periodo de retorno de 5 años & 3 \\
Periodo de retorno de 50 años & 5 \\
Periodo de retorno de 500 años & 6 \\
Fuera del modelo & 3 \\
\hline
\end{tabular}

En este caso los valores originales coinciden con los rangos de la cartografía realizada por Ollero (1996).

Tabla 4. Valores utilizados en la estandarización de la capacidad agrícola de los suelos

\begin{tabular}{|l|c|}
\hline Valor original & Valor estandarizado \\
\hline $\begin{array}{l}\text { Áreas sin suelo } \\
\text { Moderada capacidad agrícola con la deficiencia climática como } \\
\text { factor limitante }\end{array}$ & 3 \\
$\begin{array}{l}\text { Moderada capacidad agrícola con la deficiencia climática y el } \\
\text { suelo como factores limitantes } \\
\begin{array}{l}\text { Moderada capacidad agrícola con la deficiencia climática, } \\
\text { el suelo y la pendiente como factores limitantes } \\
\text { Capacidad agrícola marginal con el suelo como factor limitante }\end{array}\end{array}$ \\
\hline
\end{tabular}

Valores en función de la cartografía realizada siguiendo el modelo Cervatana (Lamelas, 2007, Lamelas et al., 2009).

Tabla 5. Valores utilizados en la estandarización de la pendiente

\begin{tabular}{|l|c|}
\hline Valor original & Valor estandarizado \\
\hline$>30 \%$ & 1 \\
$15-30 \%$ & 2 \\
$10-15 \%$ & 3 \\
$5-10 \%$ & 4 \\
$2-5 \%$ & 5 \\
$0-2 \%$ & 6 \\
\hline
\end{tabular}


Tabla 6. Valores utilizados en la estandarización de la caracterización tectónica de los suelos

\begin{tabular}{|l|c|}
\hline Valor original & Valor estandarizado \\
\hline Llanura de inundación, río, laderas sobres materiales terciarios, & 1 \\
zonas endorreicas, conos aluviales y valles de fondo plano & 3 \\
Terrazas bajas & 6 \\
Glacis y terrazas altas & \\
\hline
\end{tabular}

En este caso, los valores de preferencia de los criterios se han asignado tras entablar varias conversaciones con expertos en el tema del Ayuntamiento de Zaragoza y la Confederación Hidrográfica del Ebro. Los valores de preferencia más elevados (y por lo tanto los mayores pesos) han sido asignados a las zonas protegidas (valor $0.389=39 \%$ ), seguido por la susceptibilidad a la formación de dolinas (valor $0.249=25 \%$ ) y el riesgo de inundación (valor $0.177=17 \%$ ). Los últimos factores en importancia son la pendiente (valor $0.032=3 \%$ ) y la caracterización geotectónica (valor $0.053=5 \%$ ), ya que éstos se consideran menos importantes ya que pueden ser evitados mediante el empleo de nuevas técnicas constructivas (tabla 7). El ratio de consistencia obtenido en la asignación de pesos fue de 0,023, que está muy por debajo del valor 0,1 recomendado.

Tabla 7. Matriz de comparación por pares y pesos de los criterios para el análisis de localización óptima de zonas de uso residencial

\begin{tabular}{|l|c|c|c|c|c|c|c|}
\hline Matriz de preferencias & $A$ & $B$ & $C$ & $D$ & $E$ & $F$ & Peso \\
\hline Susceptibilidad al desarrollo de dolinas. A & 1,00 & 2,00 & 0,50 & 3,00 & 7,00 & 4,00 & 0,249 \\
Riesgo de inundación. B & 0,50 & 1,00 & 0,33 & 2,00 & 6,00 & 5,00 & 0,177 \\
Protección de zonas naturales. C & 2,00 & 3,00 & 1,00 & 4,00 & 8,00 & 6,00 & 0,389 \\
Capacidad agrícola de los suelos. D & 0,33 & 0,50 & 0,25 & 1,00 & 4,00 & 2,00 & 0,098 \\
Porcentaje de pendiente. E & 0,14 & 0,16 & 0,12 & 0,25 & 1,00 & 0,50 & 0,032 \\
Capacidad tectónica de los suelos. F & 0,25 & 0,20 & 0,16 & 0,50 & 2,00 & 1,00 & 0,053 \\
\hline
\end{tabular}

Con respecto al método EMC aplicado en este trabajo al análisis de localización óptima de uso residencial, la principal ventaja de la metodología SAW, es su bajo grado de complejidad que la hace muy atractiva para el análisis de localización óptima. Es precisamente esta simplicidad, la que hace que la suma ponderada sea actualmente una de las técnicas más ampliamente utilizadas en casos reales (Eastman, 1997; Heywood et al., 2002; Janssen y Rietveld, 1990; Malczewski y Rinner, 2005). En esta metodología, todos los factores clasificados o estandarizados (criterios), que constituyen archivos ráster independientes, son multiplicados por sus correspondientes pesos y son sumados. 


\subsection{Análisis para la selección de alternativa de localización óptima}

El objetivo final del análisis para la selección de alternativa de localización óptima es la clasificación de las alternativas en función de su preferencia. Para llevar a cabo este análisis es necesario, por tanto, disponer previamente de unas alternativas de localización, que desde el punto de vista geométrico están representadas por polígonos. En este caso, las alternativas están representadas por cuarenta zonas clasificadas como urbanizables en el Plan General de Ordenación Urbana de Zaragoza (figura 5).

En esta aproximación no es necesaria la estandarización de los valores asignados a los criterios, ya que las alternativas son directamente comparadas por pares, en función de sus valores originales, para asignar la función de preferencia. Las restricciones y los criterios son los mismos utilizados en el análisis de localización óptima. Sin embargo, existen algunas alternativas que se localizan en zonas que están parcialmente restringidas, ya que los polígonos que las delimitan son cruzados por carreteras o vías pecuarias.

Este hecho implica la introducción en el análisis de un nuevo criterio que es la cartografía de las restricciones de uso, de manera que las zonas con restricciones de uso obtienen el valor cero, mientras que las zonas sin restricciones obtienen el valor uno. Por esta razón, se deben asignar nuevamente pesos a los criterios, ya que, como se verá más adelante, en esta metodología también es necesario asignar pesos a los criterios, para lo que la metodología AHP ha sido integrada en la herramienta.

Los nuevos pesos asignados siguen las preferencias comentadas con anterioridad, aunque el mayor peso ha de ser asignado a la cartografía de restricciones para intentar seleccionar las alternativas que se localicen en zonas con restricciones de uso (ver tabla 8).

Tabla 8. Comparación de pesos asignados a los criterios en el análisis de localización óptima (A) y el análisis de selección de alternativas óptimas (B)

\begin{tabular}{|l|c|c|}
\hline Variables & $A$ & $B$ \\
\hline Susceptibilidad al desarrollo de dolinas. A & 0,249 & 0.161 \\
Riesgo de inundación. B & 0,177 & 0.106 \\
Protección de zonas naturales. C & 0,389 & 0.242 \\
Capacidad agrícola de los suelos. D & 0,098 & 0.068 \\
Porcentaje de pendiente. E & 0,032 & 0.024 \\
Capacidad tectónica de los suelos. F & 0,053 & 0.043 \\
Restricciones & - & 0.356 \\
\hline
\end{tabular}


Como se ha mencionado con anterioridad, desde el punto de vista geométrico, cada alternativa es un polígono que contiene diferentes valores para un mismo criterio (pixeles en la capa ráster). Por lo tanto, es necesario seleccionar un único valor para cada alternativa.

La metodología PROMETHEE-2 utiliza la función de preferencia Pj (a,b), la cual es una función de la diferencia entre dos alternativas para un determinado criterio (Brans et al., 1986; Marinoni, 2005, Lamelas, 2007, 2009; Lamelas et al., 2010, 2012). En este trabajo se ha utilizado la función de preferencia "usual criterion" que se basa en la simple diferencia en los valores asignados a las alternativas.

La comparación por pares de alternativas produce una matriz de preferencia para cada criterio (figura 6). Una vez calculada la matriz de preferencia para cada criterio, se realiza una primera agregación, multiplicando cada valor de preferencia por un factor de peso, w, que expresa el peso o importancia de cada criterio y sumando estos productos (Lamelas et al., 2012; Marinoni, 2005).

El resultado es un índice de preferencia, П como el que se presenta en la tabla 9. La clasificación final de idoneidad de las alternativas se realiza calculando el flujo neto $\Phi$ (a1) para cada alternativa, a, que se obtiene de la resta del flujo saliente $(\Phi+)$ y entrante $(\Phi-)$. Cuanto mayor es el flujo neto, mayor es la preferencia de una alternativa sobre las demás.

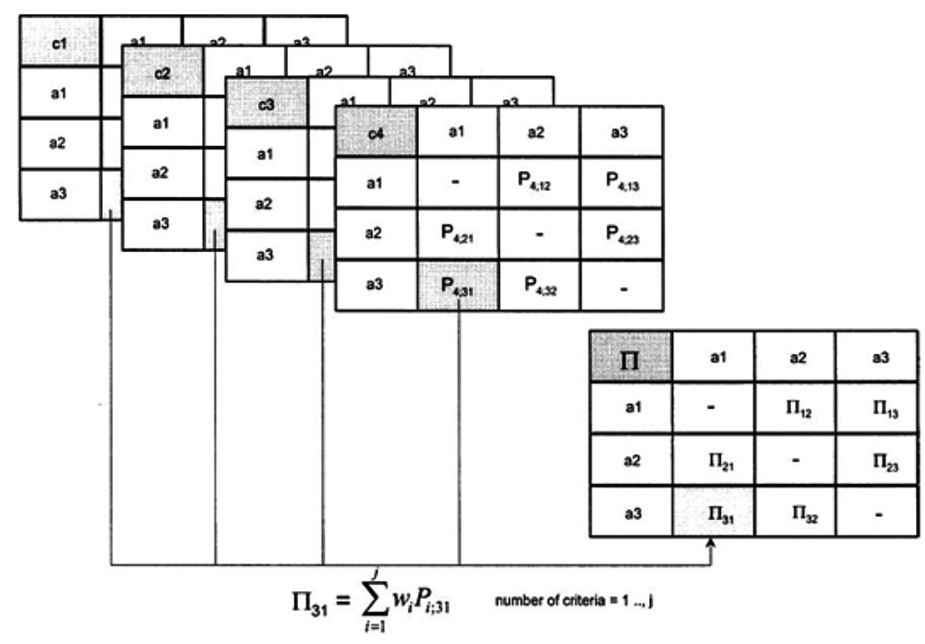

Figura 6. Esquema de cálculo del índice de preferencia.

Fuente: Marinoni (2005). 
Tabla 9. Ejemplo hipotético de índice de preferencia, flujo entrante y saliente, flujo neto y clasificación final

\begin{tabular}{|l|c|c|c|c|c|c|}
\hline & $a 1$ & $a 2$ & $a 3$ & $+(a x)$ & (ax) & Clasificación \\
\hline $\mathbf{a 1}$ & - & 0.25 & 0.75 & 1.0 & 0 & 2 \\
$\mathbf{a 2}$ & 0.75 & - & 0.75 & 1.5 & 1 & 1 \\
$\mathbf{a 3}$ & 0.25 & 0.25 & - & 0.5 & -1 & 3 \\
$-(\mathbf{a x})$ & 1 & 0.5 & 1.5 & & & \\
\hline
\end{tabular}

\subsubsection{Control de la incertidumbre: PROMETHEE-2 estocástico}

La elección de los valores de entrada en un procedimiento de análisis multicriterio no es siempre sencilla, ya que los valores de los criterios para cada una de las diferentes alternativas no siempre son únicos, como en el caso que nos ocupa, sino que pueden presentar un rango de valores (Marinoni, 2005).

En nuestro caso, el valor asignado a cada alternativa para cada criterio ha sido el valor medio de los presentes dentro de cada polígono que representa una alternativa. No obstante, al realizar el análisis utilizando el valor medio de los datos, la incertidumbre en la precisión tanto de los valores de entrada como de los resultados obtenidos es muy elevada (Lamelas, 2007; Lamelas et al., 2012).

Una posible solución consiste en llevar a cabo una aproximación estocástica que utilice distribuciones de probabilidad para generar los valores de entrada en el análisis, en lugar de utilizar un valor único. Los modelos de distribución se infieren de las poblaciones resultantes de los valores de los criterios (valores de los pixeles) de cada una de las alternativas (polígonos). El programa utilizado para ajustar los tipos de distribución, en función del test de ajuste más adecuado, ha sido @Risk (Palisade Corporation, 2002). Esta aproximación, utiliza todo el rango de posibles valores de los criterios y los casos extremos son representados como eventos raros de forma más realista, de acuerdo con su baja probabilidad de aparición.

La distribución de un criterio es muy probable que sea diferente para cada una de las alternativas debido a la variabilidad local/regional. De modo que, si los análisis estadísticos indican que existen diferentes distribuciones para un criterio en las diferentes alternativas, deben asignarse diferentes distribuciones a un mismo criterio en las distintas alternativas (Marinoni, 2005).

Es importante que las distribuciones se ajusten a las propiedades de los datos, de manera que, si los datos no admiten valores negativos (p.ej. porcentaje de pendiente) esta propiedad quede reflejada, por ejemplo seleccionando una distribución expo- 
nencial. Este es el caso de algunas alternativas en el criterio correspondiente a la susceptibilidad al desarrollo de dolinas.

El tipo de distribución utilizada más habitualmente, en el estudio que nos ocupa, para variables continuas, como la pendiente, es la distribución log-normal. No obstante, también se han utilizado las distribuciones normal, logística y exponencial, en el caso de variables continuas como la susceptibilidad al desarrollo de dolinas.

En el caso de variables categóricas con dos posibles realidades, se ha utilizado la distribución binomial. Cuando la variable categórica presentaba más de dos clases, la utilización de una distribución categórica podría dar problemas, al incluir en el análisis valores que no están presentes en la realidad. Por ejemplo, si una alternativa presenta valores 1 y 4 en el criterio correspondiente a capacidad agrícola y asignamos una distribución categórica, la simulación habría asignado valores 2 y 3 a la alternativa, aunque estos valores no están presentes en la realidad. Por esta razón, en lugar de asignar una distribución, en estos casos, se ha utilizado el porcentaje de casos en cada categoría para calcular la probabilidad de ocurrencia de cada categoría. Finalmente, algunas alternativas presentan un único valor en todos los pixeles que componen la alternativa.

Esta aproximación requiere la repetición del análisis, múltiples veces, con diferentes valores de entrada para los criterios. Con este propósito, se aplicó la simulación Monte Carlo (MCS) con un número de iteraciones n de 5.000, produciendo n valores a introducir en la matriz de entrada de datos (Lamelas, 2007; Lamelas et al., 2012).

El resultado se utiliza entonces para conocer las clasificaciones obtenidas por una determinada alternativa o bien analizar las alternativas que han obtenido una determinada clasificación. Sin embargo, la alternativa que obtiene el primer puesto en la clasificación en la mayoría de repeticiones puede no ser necesariamente la mejor. Por esta razón, se sugiere calcular el índice de clasificación estocástico SI (Lamelas, 2007; Lamelas et al., 2012; Marinoni, 2005) que cuanto más próximo a 0 se encuentra, indica una mayor idoneidad de la alternativa.

\section{Resultados}

En general, la validación de un modelo consiste en comprobar si la estructura del modelo es adecuada para obtener el fin perseguido y con él se consigue un nivel aceptable de precisión en las predicciones. En el caso de modelos explicativos o predictivos, normalmente esta validación se lleva a cabo comprobando el grado de acuerdo entre los datos arrojados por el modelo y los datos del sistema real (Gómez-Delgado, y Barredo, 2005). Para el caso del análisis de capacidad del terreno para acoger un uso residencial mediante la aplicación de SDSS, el modelo se ha validado mediante la com- 
probación de que el mismo sigue las preferencias seleccionadas y aplicadas mediante la asignación de los pesos de los criterios.

La figura 5 muestra el resultado final del análisis de búsqueda de localización óptima para uso residencial. Según el modelo desarrollado, las zonas más idóneas para la localización de zonas de uso residencial, son las terrazas y glacis al sur y sur-oeste de Zaragoza, así como algunos sectores en los valles de los ríos Jalón y Huerva y el noreste de la plataforma estructural de La Muela. Se trata de sectores en los que no se localizan zonas protegidas, factor al que se le ha asignado el mayor peso tras aplicar la metodología AHP. En la mayoría de los casos, coincide con zonas de baja susceptibilidad al desarrollo de dolinas y alejadas de las zonas con menor periodo de retorno de inundaciones. Presentan bajos valores de pendiente, aunque en algunos casos, como en las proximidades de La Muela, se localizan en sectores de malas características geotécnicas. No obstante, hay que recordar que a este último factor se le había asignado un menor peso, ya que las malas condiciones del terreno pueden ser subsanadas mediante el empleo de mejores técnicas constructivas. De acuerdo con los pesos asignados a los criterios, los valores peores de idoneidad se concentran en las zonas protegidas del entorno de los ríos, así como en los hábitats esteparios del sureste de la zona de estudio.

En la tabla 10 se presentan el flujo entrante y el flujo saliente generados por la metodología PROMETHEE-2 a partir de los índices de preferencia, así como el flujo neto que determina la clasificación final de las alternativas. Las alternativas que presentan las primeras clasificaciones y por tanto las más idóneas, considerando las preferencias de criterios establecidas, son la 40, 18, 12 y 13, que se localizan principalmente en el suroeste y sureste de la ciudad de Zaragoza (figura 7). Además, en la mayoría de los casos, las alternativas mejor clasificadas también presentan un valor medio elevado en la metodología SAW (ver figura 6), de manera que si clasificáramos las alternativas en función de ese valor medio, presentarían las clasificaciones más altas (valores SAW media y Clasif. SAW en la tabla 10).

En líneas generales, existen pocas diferencias en la clasificación realizada con la metodología PROMETHEE-2 y la versión estocástica. En la tabla 10 se presentan los valores SI y la clasificación obtenida tras aplicar la aproximación estocástica y se puede observar como las alternativas que obtienen las primeras y últimas clasificaciones, coinciden en ambas metodologías. De hecho, para medir la coincidencia entre los resultados, se ha realizado un análisis de correlación de Spearman entre los resultados, obteniendo un coeficiente de correlación de 0,78, significativo a un nivel del 0,01, lo que implica un elevado acuerdo entre los dos resultados.

No obstante, se observan algunas discrepancias entre los resultados que es interesante destacar, como es el caso del cambio en la alternativa de mayor idoneidad en función del método aplicado. Se trata de las alternativas 40 y 12 que adquieren la primera clasificación en la metodología PROMETHEE-2 y la aproximación estocástica, respectivamente. Una posible casusa es la existencia de una pequeña zona en la alternativa 12 
Tabla 10. Flujo entrante, flujo saliente, flujo neto y Clasificación PROMETHEE-2. Índice de clasificación estocástico y clasificación final estocástica.

Valor medio y clasificación de la sumatoria lineal ponderada

\begin{tabular}{|c|c|c|c|c|c|c|c|c|}
\hline Alt & + & - & & $\begin{array}{l}\text { Clasifi. } \\
\text { PROM. }\end{array}$ & $S I$ & $\begin{array}{c}\text { Clasif. } \\
\text { Estocást. }\end{array}$ & $\begin{array}{c}S A W \\
\text { media }\end{array}$ & $\begin{array}{c}\text { Clasifi. } \\
\text { SAW }\end{array}$ \\
\hline 1 & 19,82 & 7,49 & $-12,33$ & 36 & 0,70 & 35 & 4,21 & 39 \\
\hline 2 & 16,20 & 11,63 & $-4,57$ & 26 & 0,56 & 23 & 4,44 & 34 \\
\hline 3 & 12,90 & 14,07 & 1,17 & 17 & 0,54 & 20 & 4,33 & 37 \\
\hline 4 & 17,98 & 9,33 & $-8,65$ & 31 & 0,65 & 33 & 4,60 & 25 \\
\hline 5 & 17,49 & 9,83 & $-7,66$ & 30 & 0,64 & 31 & 4,66 & 23 \\
\hline 6 & 12,27 & 15,04 & 2,77 & 16 & 0,42 & 13 & 4,77 & 18 \\
\hline 7 & 14,25 & 13,07 & $-1,18$ & 21 & 0,61 & 26 & 4,49 & 28 \\
\hline 8 & 14,21 & 13,10 & $-1,11$ & 20 & 0,59 & 25 & 4,28 & 38 \\
\hline 9 & 19,86 & 7,39 & $-12,48$ & 37 & 0,78 & 38 & 4,16 & 40 \\
\hline 10 & 11,06 & 16,43 & 5,38 & 14 & 0,42 & 14 & 5,18 & 9 \\
\hline 11 & 8,32 & 19,51 & 11,19 & 8 & 0,35 & 9 & 5,35 & 5 \\
\hline 12 & 9,99 & 25,82 & 15,83 & 3 & 0,08 & 1 & 5,54 & 2 \\
\hline 13 & 10,61 & 25,20 & 14,59 & 4 & 0,08 & 2 & 5,56 & 1 \\
\hline 14 & 21,10 & 14,55 & $-6,55$ & 29 & 0,40 & 11 & 4,93 & 15 \\
\hline 15 & 9,13 & 18,70 & 9,58 & 9 & 0,26 & 5 & 5,13 & 11 \\
\hline 16 & 25,52 & 10,29 & $-15,24$ & 38 & 0,40 & 12 & 5,01 & 13 \\
\hline 17 & 11,56 & 24,09 & 12,53 & 6 & 0,13 & 3 & 5,34 & 6 \\
\hline 18 & 5,43 & 22,06 & 16,63 & 2 & 0,32 & 8 & 4,99 & 14 \\
\hline 19 & 19,57 & 7,74 & $-11,83$ & 35 & 0,78 & 39 & 4,41 & 36 \\
\hline 20 & 15,48 & 11,83 & $-3,65$ & 25 & 0,48 & 18 & 4,74 & 21 \\
\hline 21 & 13,58 & 13,91 & 0,33 & 18 & 0,44 & 15 & 4,84 & 16 \\
\hline 22 & 7,46 & 20,03 & 12,57 & 5 & 0,38 & 10 & 4,75 & 20 \\
\hline 23 & 16,61 & 10,92 & $-5,69$ & 27 & 0,72 & 37 & 5,19 & 8 \\
\hline 24 & 9,89 & 21,14 & 11,25 & 7 & 0,47 & 17 & 4,61 & 24 \\
\hline 25 & 21,08 & 9,57 & $-11,50$ & 34 & 0,68 & 34 & 4,46 & 30 \\
\hline 26 & 15,29 & 15,43 & 0,14 & 19 & 0,62 & 27 & 4,46 & 31 \\
\hline 27 & 27,71 & 10,88 & $-16,84$ & 40 & 0,63 & 30 & 4,82 & 17 \\
\hline 28 & 19,89 & 10,62 & $-9,27$ & 32 & 0,56 & 22 & 4,76 & 19 \\
\hline 29 & 9,21 & 17,95 & 8,74 & 11 & 0,31 & 7 & 5,15 & 10 \\
\hline 30 & 13,08 & 17,37 & 4,30 & 15 & 0,55 & 21 & 4,45 & 32 \\
\hline 31 & 16,42 & 14,19 & $-2,23$ & 23 & 0,70 & 36 & 4,44 & 33 \\
\hline 32 & 16,86 & 13,75 & $-3,11$ & 24 & 0,64 & 32 & 4,54 & 26 \\
\hline 33 & 10,92 & 19,80 & 8,88 & 10 & 0,58 & 24 & 4,48 & 29 \\
\hline 34 & 14,63 & 13,20 & $-1,43$ & 22 & 0,51 & 19 & 4,54 & 27 \\
\hline 35 & 21,87 & 5,44 & $-16,43$ & 39 & 0,88 & 40 & 4,43 & 35 \\
\hline 36 & 10,36 & 17,47 & 7,11 & 13 & 0,44 & 16 & 4,72 & 22 \\
\hline 37 & 19,38 & 8,45 & $-10,92$ & 33 & 0,62 & 28 & 5,38 & 3 \\
\hline 38 & 16,80 & 11,03 & $-5,77$ & 28 & 0,63 & 29 & 5,30 & 7 \\
\hline 39 & 9,70 & 18,13 & 8,42 & 12 & 0,19 & 4 & 5,37 & 4 \\
\hline 40 & 5,22 & 22,27 & 17,05 & 1 & 0,26 & 6 & 5,08 & 12 \\
\hline
\end{tabular}


que presenta bajos valores de idoneidad en los diferentes criterios, reduciendo de esta manera el valor medio incluido en la metodología PROMETHEE-2 y como consecuencia su clasificación. La reducida dimensión de la zona hace que en la aproximación estocástica estos valores se localicen en las colas de la distribución, presentando bajos valores de probabilidad de ocurrencia y al repetir el análisis 5.000 veces quedan reducidos.

Otro caso a destacar, es el de la alternativa que presenta una menor idoneidad en la metodología PROMETHEE-2, alternativa 27, y que incrementa considerablemente su posición en la clasificación al aplicar la aproximación estocástica. Esto es debido a la localización de una parte de la alternativa 27 en zona con restricción de uso, que hace que, al utilizar el valor medio en la aproximación PROMETHEE-2, el valor incluido en el análisis para este criterio sea mayor que 0, por lo tanto la alternativa es menos idónea que cualquier alternativa que no incluya zonas con restricciones de uso que obtendría el valor 0 . Por el contrario, en la aproximación estocástica al utilizar la probabilidad y debido a que el porcentaje de valores 0 (no restricciones de uso) es muy superior al de valores 1 (existencia de restricciones), y a que el resto de criterios son mejores esta alternativa presenta una mejor clasificación.

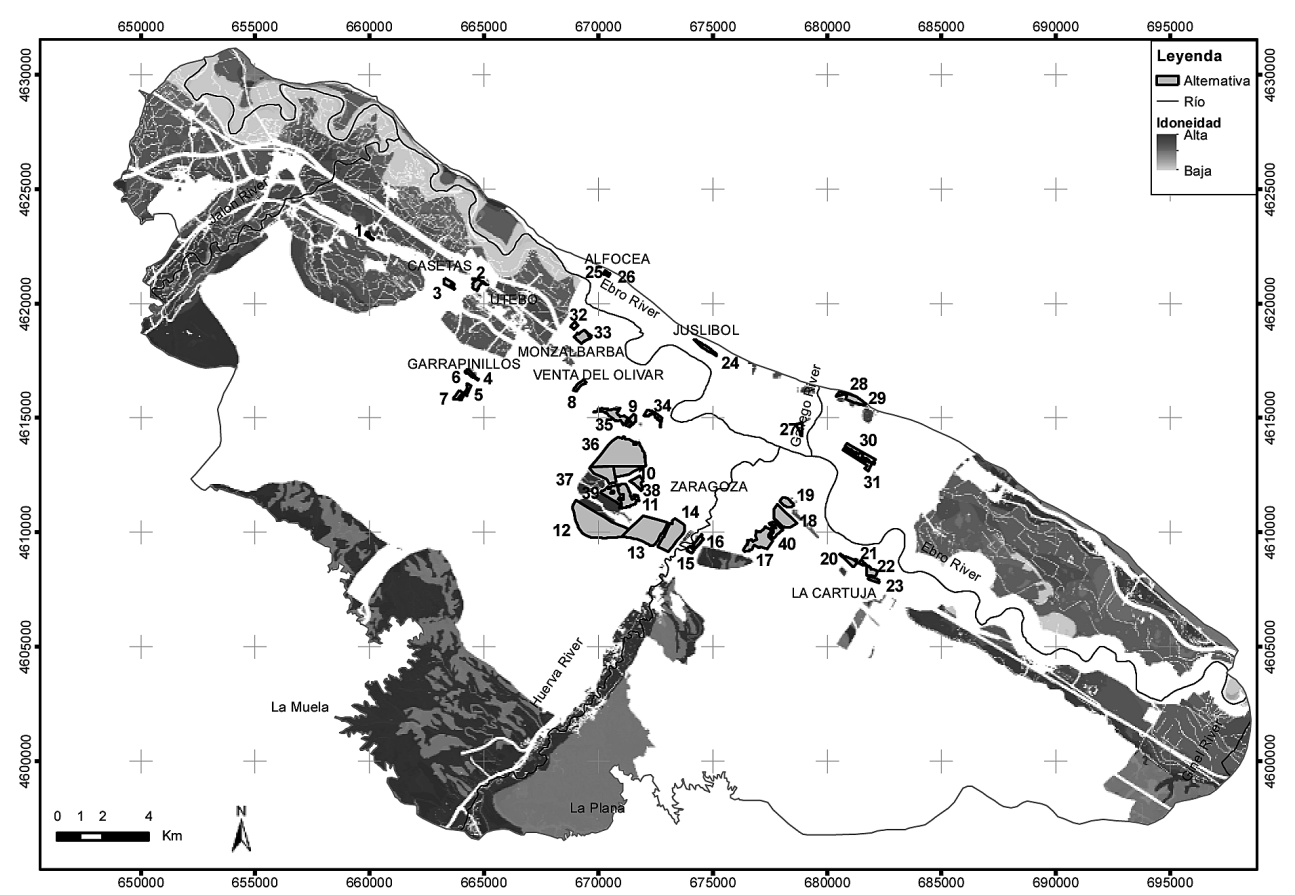

Figura 7. Alternativas incluidas en el análisis para la selección de alternativa de localización óptima superpuestas al resultado del análisis de búsqueda de localización óptima. 


\section{Discusión y conclusiones}

El objetivo final del proyecto en el que se inserta este trabajo es la integración de aspectos como los georriesgos y los georrecursos en el proceso de toma de decisiones sobre diferentes formas de uso del suelo, en grandes ciudades en expansión y crecimiento y en un medio semiárido, como es el caso de los alrededores de Zaragoza. Este objetivo se ha cubierto parcialmente en este artículo, mediante el análisis de localización óptima de zonas residenciales, a partir de la integración de diversas metodologías de EMC en SIG, quedando patente, una vez más, la utilidad de los SDSS en el proceso de toma de decisiones sobre los usos del suelo, considerando aspectos geocientíficos, tal y como es demandado por la Agenda 21.

La metodología AHP, utilizada en este artículo, es un excelente y ampliamente utilizado método de EMC, diseñado para resolver uno de los problemas fundamentales en la teoría de la decisión; el cómo asignar pesos a un conjunto de criterios de acuerdo con su importancia. Esta metodología trata de resolver de la forma más objetiva posible, el problema de la subjetividad inherente a la toma de decisiones sobre los usos del suelo por parte de los agentes de decisión.

Además, para reforzar la objetividad en la asignación de los pesos a los criterios, así como incluir la participación pública en el análisis, los pesos de los criterios se asignaron tras entablar varias conversaciones con expertos en el tema del Ayuntamiento de Zaragoza y la Confederación Hidrográfica del Ebro. Finalmente, teniendo en cuenta que el objetivo final es el desarrollo sostenible, las zonas más idóneas para la localización de zonas de uso residencial, son las terrazas y glacis al sur y sur-oeste de Zaragoza, así como algunos sectores en los valles de los ríos Jalón y Huerva y el noreste de La Muela. Se trata de sectores en los que no se localizan zonas protegidas, factor al que se le ha asignado el mayor peso tras aplicar la metodología AHP. En la mayoría de los casos, coincide con zonas de baja susceptibilidad al desarrollo de dolinas y alejadas de las zonas con menor periodo de retorno de inundaciones.

En el problema de selección de alternativa óptima, la existencia de un número relativamente reducido de alternativas, posibilita la utilización de métodos de concordancia como PROMETHEE-2 que presentan la ventaja de no requerir la estandarización de las variables, lo que en definitiva reduce incluso más la subjetividad. Sin embargo, en un análisis espacial de EMC existen varias decisiones que deben ser tomadas, como, por ejemplo, que valor de los presentes en la alternativa escoger, de entre la población de pixeles que la conforman, para llevar a cabo el análisis posterior (p.ej. máximo, mínimo, media, etc.). La aproximación estocástica utilizada en este trabajo, ayuda a resolver este problema mediante la asignación de distribuciones de probabilidad a los valores de entrada, en lugar de un único valor.

Es de resaltar la similitud existente entre los resultados obtenidos en el análisis de localización óptima y los resultados del análisis de alternativa óptima. En general, las 
alternativas con mejores clasificaciones en el análisis de alternativas óptimas se localizan en las zonas con valores de mayor idoneidad en el análisis de localización óptima.

En líneas generales, existen pocas diferencias en la clasificación realizada con la metodología PROMETHEE-2 y la versión estocástica. Las alternativas que obtienen las primeras y últimas clasificaciones coinciden en ambas metodologías. No obstante, se observan algunas discrepancias entre los resultados, principalmente derivadas del hecho de que la aproximación estocástica tiene en cuenta la probabilidad de ocurrencia de un valor de un determinado criterio en una alternativa, reduciendo, de este modo, la incertidumbre existente en la precisión, tanto de los valores de entrada, como de los resultados obtenidos.

Por este motivo, es nuestra recomendación el utilizar aproximaciones estocásticas cuando se trabaja en un entorno de análisis multicriterio de datos y variables espaciales, aunque, en este caso, el proceso no esté absolutamente integrado en el SIG, y por lo tanto sea más tedioso.

\section{Agradecimientos}

Esta investigación ha sido posible gracias a la subvención del Servicio de Investigación Alemán (DFG, Ho 804/7-1+2) y a la colaboración del Departamento de Geografía y Ordenación del Territorio de la Universidad de Zaragoza. Es necesario dar las gracias a los directores y principal colaborador de este trabajo, Prof. Dr. Andreas Hoppe, Dr. Juan de la Riva y Oswald Marinoni por sus aportaciones en la investigación. Nos gustaría dar las gracias a la Confederación Hidrográfica del Ebro, el Gobierno de Aragón, el Ayuntamiento de Zaragoza, por la información que nos proporcionaron.

\section{Bibliografía}

Amadio, V., Amadei, M., Bagnaia, R., Di Bucci, D., Laureti, L., Lisi, A., Lugeri, F.R., Lugeri, N. (2002) The role of geomorphology in Landscape Ecology: the Landscape Unit Map of Italy, Scale 1:250,000 (Carta Della Natura Project). En Allison, R.J. (ed) Applied Geomorphology: theory and Practice. Chichester, John Wiley and Sons Ltd.

Banai, R. (1993) Fuzziness in geographical information systems: contributions from the analytic hierarchy process. International
Journal Geographical Information Systems, 7(4), 315-329.

Banai-kashani, R. (1989) A new method for site suitability analysis: the analytical hierarchy process. Environmental Management, 13, 685-693.

Barredo, J. y Bosque, J. (1995) Integración de evaluación multicriterio y sistemas de información geográfica para la evaluación de la capacidad de acogida del territorio y 
la asignación de usos del suelo. Actas del IV Congreso Español de Sistemas de información Geográfica, 191-200, Barcelona, AESIG.

Bosque, J. y García, R.C. (2000): El uso de los sistemas de información geográfica en la planificación territorial. Anales de Geografía de la Universidad Complutense, 20, 49-67.

Bosque, J. y Moreno, A. (2004) Sistemas de información geográfica y localización óptima de instalaciones y equipamientos. Madrid, RA-MA.

Bosque, J., Diaz, M.A., Gómez, M., Rodríguez, V.M., Rodríguez, A.E. y Vela, A. (1999) Un procedimiento basado en un SIG, para localizar centros de tratamiento de residuos. Anales de Geografía de la Universidad Complutense, 9, 295-323.

C.S.I.C. (1996) Microleis 4.1: Exploring the Agro-ecological Limits of Sustainability. ProEEco. Agro-ecological Land Quality Evaluation System. Sevilla, Instituto de Recursos Naturales y Agrobiología.

Can, A. (1993) Residential quality assessment, Alternative approaches using GIS. En Fischer, M. y Nijkamp, P. (eds) Geographic information systems, spatial modelling and policy evaluation. Berlin, Springer-Verlag.

Carver, S.J. (1991) Integrating multi-criteria evaluation with geographical information systems. International Journal of Geographical Information Systems, 5, 321-339.

de la Rosa, D. y Magaldi, D. (1982) Rasgos metodológicos de un sistema de evaluación de tierras para regiones mediterráneas. Madrid, Sociedad Española de la Ciencia del Suelo.

de la Rosa, D., Mayol, F., Moreno, F., Cabrera, F., Díaz-Pereira, E. y Antoine, J. (2002) A multilingual soil profile database (SDBm Plus) as an essential part of land resources information Systems. Environmental Modelling \& Software, 17, 721-730. de la Rosa, D., Mayol, F., Díaz-Pereira, E., Fernández, M. y de la Rosa Jr, D. (2004) A land evaluation decision support system (MicroLEIS DSS) for agricultural soil protection with especial reference to the Mediterranean region. Environmental Modelling $\mathcal{E}$ Software, 19, 929-942.

Eastman, J.R. (1997) IDRISI for Windows, Version 2.0: Tutorial exercises. Worcester, Graduated School of Geography. Clark Unversity.

Eastman, J.R., Kyem, P.A., Toledano, J. y Jin, W. (1993) GIS and Decision Making. Ginebra, United Nations institute for Training and Research.

Gómez-Delgado, M. y Barredo, J.I. (2005) Sistemas de Información Geográfica y evaluación multicriterio en la ordenación del territorio. Paracuellos del Jarama, Editorial RA-MA.

Heywood, I., Cornelius, S. y Carver, S. (2002) An introduction to geographical information Systems. Harlow, Prentice Hall.

Jankowski, P. (1995) Integrating geographical information systems and multiple criteria decision-making. International Journal of $\mathrm{Ge}$ ographical Information Systems, 9, 251-273.

Janssen, R. y Rietveld, P. (1990) Multicriteria analysis and GIS: An Application to Agricultural Land-use in the Netherlands. En Scholten, H y Stillwell, J. (eds) Geographical Information Systems for Urban and Regional Planning. Dordrecht. Kluwer.

Lamelas, M.T. (2007) Geo-resources and geohazards in the context of a sustainable development in the periphery of urban areas, exemplary of a part of the Ebro Basin in the surroundings of Zaragoza (Spain). Tesis doctoral. Technische Universität Darmstadt. Disponible en http://elib.tu-darmstadt.de/diss/000794 (último acceso Abril de 2013). 
Lamelas, M.T. (2007b) Análisis y cartografía mediante SIG de la susceptibilidad a la formación de dolinas en el entorno de Zaragoza. Geographicalia, 52, 79-111.

Lamelas, M.T. (2009) Esquema metodológico para la toma de decisiones sobre el uso sostenible del suelo: Aplicación a la localización de suelo industrial. GeoFocus, 9, 28-66.

Lamelas, M.T., Marinoni, O., de la Riva, J. y Hoppe, A. (2006) The use of Spatial Decision Support Systems for sand and gravel extraction suitability in the context of a sustainable development in the surroundings of Zaragoza (Spain). En Instituto Cartográfico de Cataluña (ed.). Proceedings of the 5th European Congress on Regional Geoscientific Cartography and Information Systems. 180-183, Barcelona.

Lamelas, M.T., Marinoni, O., Hoppe, A. y de la Riva, J. (2007) Groundwater vulnerability map for the Ebro alluvial aquifer between Jalón and Ginel tributaries (Spain). Environmental Geology, 53, 861-878.

Lamelas, M.T., Marinoni, O., Hoppe, A. y de la Riva, J. (2008a) Suitability analysis for sand and gravel extraction site location in the context of a sustainable development in the surroundings of Zaragoza (Spain). Environmental Geology, 55(8), 1673-1686.

Lamelas, M.T., Marinoni, O., Hoppe, A. y de la Riva, J. (2008b) Dolines probability map using logistic regression and GIS technology in the central Ebro Basin (Spain). Environmental Geology, 54, 963-977.

Lamelas, M.T., Marinoni, O., Hoppe, A. y de la Riva, J. (2009) Modelling environmental variables for geohazards and georesources assessment to support sustainable land-use decisions in Zaragoza (Spain). Geomorphology, 111, 88-103.

Lamelas, M.T., Marinoni, O., de la Riva, J. y Hoppe, A. (2010) Sustainable spatial decision making in a complex $3 \mathrm{~d}$ environment: an auditable decision support workflow based on multicriteria analysis. En Sanders M.H. y Clark Ph.D. (eds.). Geomorphology: Processes, Taxonomy and Applications (Series: Earth Sciences in the 21st Century), 144, New York, Nova Science Publishers.

Lamelas, M.T., Marinoni, O., de la Riva, J. y Hoppe, A. (2012) Comparison of Multicriteria Analysis Techniques for Environmental Decision Making on Industrial Location. En Chiang, J. (ed). Decision Support Systems. 197-222, Rijeka, InTech.

Machín, J. y Navas, A. (1998) Spatial analysis of gypsiferous soils in the Zaragoza province (Spain), using GIS as an aid to conservation. Geoderma, 87: 57-66.

Malczewski, J. (1999) GIS and multicriteria decision analysis. New York, Wiley.

Malczewski, J. (2004) GIS-based land-use suitability analysis: a critical overview. Progress in Planning, 62, 3-65.

Malczewski, J. y Rinner, C. (2005) Exploring multicriteria decision strategies in GIS with linguistic quantifiers: A case study of residential quality evaluation. Journal of Geographical Systems, 7, 249-268.

Malczewski, J., Moreno-Sánchez, R., Bojorquez-Tapia, L.A. y Ongay-Delhumeau, E. (1997) Environmental conflict analysis in the Cape Region, Mexico. Journal of Environmental Planning and Management, 40(3), 349-374.

Marinoni, O. (2004) Implementation of the analytical hierarchy process with VBA in ArcGIS. Computers and Geosciences, 30, 637-646.

Marinoni, O. (2005) A stochastic spatial decision support system based on PROMETHEE. International Journal of Geographical Information Science, 19(1), 51-68.

McGranahan, G. y Satterthwaite, D. (2003) Urban centers: An assessment of Sustainabi- 
lity. En Matson, P., Gadgil, A. y Kammen, D.M. (ed) Annual Review of Environment and Resources. 243-274, Palo Alto.

Miller, L., Collins, M.G., Steiner, F.R. y Cook, E. (1998) An approach for greenway suitability analysis. Landscape and Urban Planning, 42, 91-105.

Mitlin, D. y Satterthwaite, D. (1996) Sustainable Development and Cities. En Pugh, C. (ed). Sustainability, the Environment and Urbanization. 23-64, London, Earthscan publications Limited.

Moreno, A. y Bosque, J. (2010) Los modelos de localización óptima como herramientas para la planificación territorial y urbana de instalaciones y equipamientos. Ciudad y territorio. Estudios territoriales. 165-166, 461-480.

Ollero, A. (1996) El curso medio del Ebro. Zaragoza, Consejo de Protección de la Naturaleza de Aragón.

Palisade Corporation (2002) @ Risk 4.5.2. Advanced risk analysis for spreadsheets. New York, Newfield.
Pereira, J.M.C. y Duckstein, L. (1993) A multiple criteria decision-making approach to GIS-based land suitability evaluation. International Journal of Geographical Information Systems, 7, 407-424.

Soriano, M.A. y Simón, J.L. (1995) Alluvial dolines in the central Ebro basin, Spain: a spatial and developmental hazard analysis. Geomorphology, 11, 295-309.

Thill, J.C. (1999) Spatial Multicriteria Decision Making and Analysis. A geographic information science approach. Ashgate, Aldershot.

Vincke, P. (1986) Analysis of multicriteria decision aid in Europe. European Journal of Operational Research, 25, 160-168.

Voogd, H. (1983) Multicriteria evaluation for urban and regional planning. London, Pion Limited. 